\title{
Revisiting the ADT mass of the five-dimensional rotating black holes with squashed horizons
}

\author{
Jun-Jin Peng ${ }^{\mathrm{a}}$ \\ Guizhou Provincial Key Laboratory of Radio Astronomy and Data Processing, Guizhou Normal University, 550001 Guiyang, Guizhou, \\ People's Republic of China
}

Received: 22 July 2017 / Accepted: 9 October 2017 / Published online: 25 October 2017

(C) The Author(s) 2017. This article is an open access publication

\begin{abstract}
We evaluate the Abbott-Deser-Tekin (ADT) mass of the five-dimensional rotating black holes with squashed horizons on two different on-shell reference backgrounds, which are the flat background and the boundary matched Kaluza-Klein (KK) monopole. The mass on the former, identified with the one on the background of the asymptotic geometry, differs from the mass on the latter by that of the KK monopole. However, each mass satisfies the first law of black hole thermodynamics. To test the results in five dimensions, we compute the mass in the context of the dimensionally reduced theory. Finally, in contrast with the original ADT formulation, its off-shell generalisation is applied to calculate the mass as well.
\end{abstract}

\section{Introduction}

Over the past few years, a special class of black hole solutions endowed with squashed horizons in five dimensions have been constructed since Ishihara and Matsuno presented the static charged one in the context of the Einstein-Maxwell theory [1]. Henceforth the Ishihara-Matsuno solution was generalised to the theory of five-dimensional Einstein-Maxwelldilaton gravity [2]. Apart from these static solutions, by virtue of the so-called squashing transformation generated by a function depending only on the radial coordinate, the rotating black hole solution with squashed horizons in Einstein gravity [3] and the charged (rotating) ones in the frameworks of five-dimensional supergravity [4-7] as well as EinsteinMaxwell-dilaton gravity [8] were found in succession. For other relevant solutions see $[9,10]$. Such a class of black holes possess the universal asymptotical structure, which is the same as the one of the Kaluza-Klein (KK) magnetic monopole, that is, a twisted $S^{1}$ fiber bundle over the two-sphere $S^{2}$ in a four-dimensional Minkowski spacetime.

a e-mail: pengjjph@163.com
Moreover, the existence of the compact direction allows for the possibility to perform KK dimensional reduction to them. Doing so, one will find that they turn into exact solutions of the field equations in the dimensionally reduced theories. Because of such similar features appearing in KK theory, the black holes with squashed horizons are sometimes referred to as the squashed KK black holes in the literature.

Thanks to the universal asymptotic structure arising from the squashed transformation, the squashed KK black holes exhibit some very interesting properties in contrast with their un-squashed counterparts, which can be partially found in Refs. [11-22]. As a prominent one of them, these black holes have provided opportunities to test several approaches on conserved quantities for various gravity theories, such as the Komar integral, the ADM formalism, the counterterm subtraction method, the (off-shell generalised) Abbott-DeserTekin (ADT) formulation and so on [2-4,8,23-31]. For the sake of better understanding the conserved charges of the squashed KK black holes, we are going to focus on their charges in terms of the ADT formalism [32-36] later on.

As is well known, in the framework of the original ADT formulation, it is assumed that the gravitational field is linearised around a fixed reference background that is onshell (i.e. the solution for the Euler-Lagrange equation of motion). This indicates that such a formalism is backgrounddependent. As a consequence, in order to obtain physically meaningful conserved charges, it is of significant importance to seek for reference backgrounds with appropriate matching conditions. For the black holes with squashed horizons, as demonstrated in the literature [2,3,23-28], the most popular reference backgrounds adopted to evaluate their conserved charges are the off-shell asymptotic geometries, which are not exact solutions of the field equations. However, strictly speaking, to fully comply with the spirit of the original ADT formalism, we desire the reference backgrounds to satisfy the equations of motion. Therefore, apart from the asymptotic 
structures of the squashed KK black holes, it is very necessary to find other on-shell reference backgrounds rendering the original ADT method successful in the computation of their charges.

As was already indicated in Ref. [28], there exist two onshell reference backgrounds, namely, the flat background [25] together with the boundary matched KK monopole, which are appropriate in the evaluation of the usual ADT mass of the squashed static charged KK black holes within the theory of five-dimensional Einstein-Maxwell-dilaton gravity. Thereafter, an interesting question that naturally arises is whether both the reference backgrounds are further applicable for the more general (charged) rotating black holes with squashed horizons [3-8], although the mass of the rotating ones has been evaluated on the usual off-shell asymptotic geometry in $[3,26]$. If so, we are able to provide universal on-shell reference backgrounds to make the usual ADT formulation yield physical results of all the squashed KK black holes.

Inspired by Ref. [28], our purpose here is to look for onshell reference backgrounds instead of the off-shell asymptotic structure in [3,26], on which the usual ADT mass of the squashed rotating KK black holes [3] can be computed. As we shall see below, the flat background and the KK monopole adopted in the static cases also hold for these rotating black holes through a few modifications. Besides, to examine the five-dimensional results, we modify the usual ADT formulation by including the contributions from gauge fields as well as scalars and then deal with the mass on two reference spacetimes in the context of the four-dimensional reduced theory. Finally, as a comparison, the off-shell generalisation of the usual ADT formalism [37] is applied to calculation of the mass as well. It should be emphasised that both reference spacetimes can also be adopted to evaluate the ADT angular momentum of the squashed rotating black holes, although we merely take into consideration of their mass.

The outline of this work is as follows. In Sect. 2, the mass of the squashed rotating KK black holes is computed on the flat background in the framework of the usual ADT formalism. In Sect. 3, the mass of these black holes is evaluated on the boundary matched KK monopole. In Sect. 4, the mass in five dimensions is examined in the context of the fourdimensional reduced theory. In comparison with the original ADT formalism, its off-shell generalisation is applied to calculate the mass of these black holes in Sect. 5. The last section is for our conclusions.

\section{ADT mass on the flat background and the first law of black hole thermodynamics}

In this section, our main goal is to apply the conventional ADT formalism to calculate the mass of the rotating black holes with squashed horizons in the theory of Einstein gravity on the flat reference background. The solution for these black holes was first found by Dobiasch and Maison [49-51] and it was regenerated via performing the squashing transformation to the solution of the usual five-dimensional rotating black holes with two equal angular momenta by Wang [3]. For the concrete relation between the two forms of the solutions see Ref. [29].

For the neutral squashed rotating KK black holes in five dimensions, their line element can be written in a general form as follows [3]:

$$
\begin{aligned}
\mathrm{d} s_{(5)}^{2}= & -\frac{\Delta \Sigma_{i}}{\Sigma \Delta_{i}} \mathrm{~d} t^{2}+\frac{r^{2} H \mathcal{K}^{2}}{\Delta} \mathrm{d} r^{2}+\frac{H \mathcal{K}}{4}\left(\mathrm{~d} \theta^{2}+\sin ^{2} \theta \mathrm{d} \phi^{2}\right) \\
& +\frac{\Sigma}{4 H}(\mathrm{~d} \psi+\cos \theta \mathrm{d} \phi-2 m a \omega \mathrm{d} t)^{2},
\end{aligned}
$$

in which

$$
\begin{aligned}
\omega & =\sqrt{\frac{\Sigma_{i}}{\Delta_{i}}}\left(\frac{1}{\Sigma}-\frac{1}{\Sigma_{i}}\right), \\
H & =r^{2}+a^{2}, \quad \Sigma=H^{2}+m a^{2}, \quad \Delta=\Sigma-m H, \\
\Sigma_{i} & =\left.\Sigma\right|_{r=r_{\infty}}, \quad \Delta_{i}=\left.\Delta\right|_{r=r_{\infty}}, \quad \mathcal{K}=\frac{\Delta_{i}}{\left(r^{2}-r_{\infty}^{2}\right)^{2}} .
\end{aligned}
$$

In the above equations, the constant $r_{\infty}$ relates to the size of an $S^{1}$ fiber at spatial infinity, playing a pivotal role in the asymptotic behaviour of the solution, while we shall see in a moment that the two integral constants $m$ and $a$ are related to the mass and angular momentum, respectively. Besides, the Euler angles $(\theta, \phi, \psi)$ take the values $(0<\theta<\pi$, $0<\phi<2 \pi, 0<\psi<4 \pi)$, and the radial coordinate $r$ is located in the interval $\left(0, r_{\infty}\right)$. To see the asymptotic structure of the squashed rotating KK black holes, as usual, we introduce a new radial coordinate $\rho$ by

$$
\rho=\rho_{0} \frac{r^{2}}{r_{\infty}^{2}-r^{2}}, \quad \rho_{0}^{2}=\frac{\Delta_{i}}{4} \frac{r_{\infty}^{2}+a^{2}}{r_{\infty}^{4}} .
$$

Such a transformation maps the radial coordinate $r \in\left(0, r_{\infty}\right)$ into $\rho \in(0, \infty)$. Subsequently, in the $\rho \rightarrow \infty$ limit, corresponding to the situation $r \rightarrow r_{\infty}$, the metric ansatz (2.1) approaches the following form:

$$
\begin{aligned}
\mathrm{d} s_{\text {asym }}^{2}= & -\mathrm{d} t^{2}+\mathrm{d} \rho^{2}+\rho^{2}\left(\mathrm{~d} \theta^{2}+\sin ^{2} \theta \mathrm{d} \phi^{2}\right) \\
& +\frac{\tilde{r}_{\infty}^{2}}{4}(\mathrm{~d} \psi+\cos \theta \mathrm{d} \phi)^{2},
\end{aligned}
$$

where the parameter $\tilde{r}_{\infty}$, which can be seen as the radius of the compactified direction at infinity, is given by

$\tilde{r}_{\infty}^{2}=\frac{\Sigma_{i}}{H_{i}}=\frac{\left(r_{\infty}^{2}+a^{2}\right)^{2}+m a^{2}}{r_{\infty}^{2}+a^{2}}$

with $H_{i}=\left.H\right|_{r=r_{\infty}}$. The asymptotic structure (2.5) possesses the same form as the generic asymptotic topology of the squashed KK black holes, that is, a twisted $\mathrm{S}^{1}$ bundle over 
a four-dimensional Minkowski spacetime. Thus the metric (2.1) is asymptotically locally flat. In [29], a new equivalent form for the five-dimensional rotating black holes with squashed horizons was directly expressed in the coordinates $(t, \rho, \theta, \phi, \psi)$, in which the asymptotic structure (2.5) naturally emerges.

As demonstrated in Ref. [3,26], the mass of the squashed rotating KK black holes was evaluated in the ADT formulation on the reference background with the asymptotical spacetime (2.4), which is not an exact solution of the vacuum Einstein equation. That is to say, the background metric is off-shell. However, strictly speaking, the original ADT current requires that the background metric is on-shell although the potential corresponding to such a current is in agreement with the off-shell generalised one in [37-40]. Thus, in order to fully obey the spirit of the usual ADT formalism [32-36], here we shall look for an appropriate on-shell reference metric to recalculate the ADT mass of these black holes. Before doing this, we first present the definition of the ADT conserved charge related to the $D$-dimensional Einstein-Hilbert Lagrangian $\mathfrak{L}_{\mathrm{gr}}=\sqrt{-g} R$, that is,

$$
\begin{aligned}
\mathcal{Q}= & \frac{1}{16 \pi G_{N}^{(D)}(D-2) !} \int_{\partial \Sigma} \sqrt{-\bar{g}} Q_{\mathrm{gr}}^{\mu \nu} \epsilon_{\mu \nu \mu_{1} \mu_{2} \cdots \mu_{(D-2)}} d x^{\mu_{1}} \\
& \wedge \cdots \wedge d x^{\mu_{(D-2)}}
\end{aligned}
$$

where the completely antisymmetric Levi-Civita tensor density $\epsilon_{\mu \nu \mu_{1} \mu_{2} \cdots \mu_{(D-2)}}$ is defined through the equation $\epsilon_{\mu_{1} \mu_{2} \cdots \mu_{D}}=D ! \delta_{\left[\mu_{1}\right.}^{0} \delta_{\mu_{2}}^{1} \cdots \delta_{\left.\mu_{D}\right]}^{D-1}$, while $G_{N}^{(D)}$ stands for the gravitational constant in $D$ dimensions. The conventional ADT potential associated with the Killing vector $\xi^{\mu}$ is read off as

$$
\begin{aligned}
Q_{\mathrm{gr}}^{\mu \nu}= & \xi_{\sigma} \bar{\nabla}^{[\mu} h^{\nu] \sigma}-h^{\sigma[\mu} \bar{\nabla}_{\sigma} \xi^{\nu]}+\frac{1}{2} h \bar{\nabla}^{[\mu} \xi^{\nu]} \\
& -\xi^{[\mu} \bar{\nabla}_{\sigma} h^{\nu] \sigma}+\xi^{[\mu} \bar{\nabla}^{\nu]} h .
\end{aligned}
$$

Here the perturbations of the gravitational field $h_{\mu \nu}$ are defined through the divergence between the original metric $g_{\mu \nu}$ and the fixed background one $\bar{g}_{\mu \nu}$, namely, $h_{\mu \nu}=g_{\mu \nu}-$ $\bar{g}_{\mu \nu}$, rather than determined by the infinitesimal changes of the solution parameters like in the off-shell generalised ADT method [37], which inherits the way of fluctuating various fields proposed in [44-46]. In general, for the asymptotically non-rotating black holes, the timelike Killing vector associated with the mass is chosen as $\xi^{\mu}=-\delta_{t}^{\mu}$ [41].

Next, we proceed to calculate the ADT mass of the squashed rotating KK black holes on the reference metric with the line element

$$
\begin{aligned}
\mathrm{d} s_{\text {flat }}^{2}= & -\mathrm{d} t^{2}+\mathrm{d} \rho^{2}+\rho^{2}\left(\mathrm{~d} \theta^{2}+\sin ^{2} \theta \mathrm{d} \phi^{2}\right) \\
& +\frac{\tilde{r}_{\infty}^{2}}{4} \mathrm{~d} \psi^{2},
\end{aligned}
$$

which is an exact flat solution to the five-dimensional Einstein gravitational field equation with a zero matter tensor. For convenience, in the above, this reference metric has been mentioned as the flat background like in Ref. [25], where it was emphasised already that the metric (2.8) with the parameter $r_{\infty}$ instead of $\tilde{r}_{\infty}$ is appropriate for the reference background in the evaluation of the mass of the squashed static charged KK black holes. Quite recently, the same flat background was successfully applied to compute the ADT mass of the static charged dilaton black holes with squashed horizons in [28]. In fact, we demonstrate here that the metric (2.8) is applicable for the squashed rotating KK black holes as well. Further employing Eq. (2.6) to calculate the mass $M$ on the flat reference background (2.8), we get

$$
\begin{aligned}
M & =\frac{\pi}{4 G_{N}^{(5)}} \frac{\left(\Delta_{i}+2 m H_{i}\right)\left(\Sigma_{i}-2 m a^{2}\right)}{H_{i} \sqrt{\Delta_{i} \Sigma_{i}}} \\
& =\frac{\pi}{4 G_{N}^{(5)}} \frac{\tilde{r}_{s}^{2}\left(\tilde{r}_{\infty}^{2}+m\right)}{\tilde{r}_{\infty} \sqrt{\tilde{r}_{\infty}^{2}-m}},
\end{aligned}
$$

in which $\tilde{r}_{s}^{2}=\sqrt{\tilde{r}_{\infty}^{4}-4 m a^{2}}$. The mass $M$ coincides with the ADT one computed on the asymptotical spacetime (2.4) in $[3,26]$ and the mass through the counterterm method [3, 29]. Further computing the angular momentum $J_{\psi}$ along the $\psi$ direction on the flat background (2.8), we obtain $J_{\psi}=$ $\pi m a / 4$, agreeing with the one via the Komar integral.

With the help of the angular velocity $\Omega_{H}$ of the event horizon $r_{+}$, the temperature $T$ and the entropy $S$, given by

$$
\begin{aligned}
\Omega_{H} & =2 \operatorname{ma\omega }\left(r_{+}\right), \quad T=\frac{\left(r_{+}-r_{-}\right) \mathcal{K}\left(r_{-}\right)}{2 \pi H\left(r_{+}\right)} \sqrt{\frac{\Sigma_{i}}{\Delta_{i}}}, \\
S & =\frac{\pi^{2}}{2} m r_{+} \mathcal{K}\left(r_{+}\right),
\end{aligned}
$$

respectively, where the outer horizon $r_{+}$and the inner horizon $r_{-}$are read off as $r_{ \pm}^{2}=\left(m-2 a^{2} \pm \sqrt{m^{2}-4 m a^{2}}\right) / 2$, we find that the ADT mass $M$ satisfies the first law of black hole thermodynamics [26,29]

$$
\begin{aligned}
& \mathrm{d} M=T \mathrm{~d} S+\Omega_{H} \mathrm{~d} J_{\psi}+\mathcal{I} \mathrm{d} L_{\infty}, \\
& 2 M=3 T S+3 \Omega_{H} J_{\psi}+\mathcal{I} L_{\infty} .
\end{aligned}
$$

In the above equation, the gravitational tension $\mathcal{I}$ and the length $L_{\infty}$ of the compactified fifth dimension at infinity are presented by

$\mathcal{I}=\frac{1}{8 G_{N}^{(5)}} \frac{\left(\Delta_{i}+\Sigma_{i}\right)\left(\Sigma_{i}-2 m a^{2}\right)}{\Sigma_{i} \sqrt{H_{i} \Delta_{i}}}, \quad L_{\infty}=2 \pi \tilde{r}_{\infty}$,

respectively, which can be thought of as thermodynamic variables resulting from the contribution from the extra dimension of the KK-type black holes [53-57]. When $r_{\infty} \rightarrow 0$, Eq. (2.11) smoothly transforms to the usual expressions for the first law of the ordinary five-dimensional black holes with 
two equal rotations in spite of the divergence for the quantities $M, \mathcal{I}$ and $L_{\infty}$. However, it should be stressed that neither the gravitational tension $\mathcal{I}$ nor the length $L_{\infty}$ of the extra dimension can be regarded as a natural thermodynamical variable with respect to the Komar mass $M_{K}$ of the squashed rotating $\mathrm{KK}$ black holes, given by $M_{K}=M-\mathcal{I} L_{\infty} / 2[5,26]$, since $M_{K}$ fails to fulfill the differential form of the first law in Eq. (2.11) although it satisfies the integral expression $2 M_{K}=3 T S+3 \Omega_{H} J_{\psi}$. It turns out that this problem can be resolved by adopting a pair of new variables $\left(W_{\infty}, \Xi_{\infty}\right)$ like in $[24,25]$, where the natural variable $W_{\infty}$ is expressed as $W_{\infty}=\mathcal{I}\left(2 c_{\psi} L_{\infty}\right)^{-1}$ in terms of the constant $c_{\psi}$, while the conjugate one $\Xi_{\infty}$ is defined through $\Xi_{\infty}=c_{\psi} L_{\infty}^{2}$. Consequently, we obtain the first law involving the Komar mass

$\mathrm{d} M_{K}=T \mathrm{~d} S+\Omega_{H} \mathrm{~d} J_{\psi}-\Xi_{\infty} \mathrm{d} W_{\infty}$

which takes a different form from the one in Eq. (2.11). Moreover, in the light of the new variables $\left(W_{\infty}, \Xi_{\infty}\right)$, one observes that both the ADT mass and the Komar mass are associated with each other through the Legendre transformation $M=M_{K}+W_{\infty} \Xi_{\infty}$, giving rise to another differential expression for the first law $\mathrm{d} M=T \mathrm{~d} S+\Omega_{H} \mathrm{~d} J_{\psi}+W_{\infty} d \Xi_{\infty}$ relative to $M$. This further demonstrates that the first law associated with the ADT mass is non-unique.

\section{ADT mass in terms of the KK monopole background and the comparison between different masses}

Besides the flat background in the previous section, as we will see shortly, the boundary matched KK monopole is appropriate for being the reference background in the evaluation of the ADT mass of the rotating black holes with squashed horizons in this section.

To seek another suitable background spacetime that we anticipates, we now solve Eq. (2.5) to obtain

$r_{\infty}^{2}=\frac{1}{2}\left(\tilde{r}_{\infty}^{2}+\tilde{r}_{s}^{2}-2 a^{2}\right)$

Substituting the above equation into the quantities $\Sigma_{i}, \Delta_{i}$ and $\mathcal{K}$ given by Eq. (2.2) to replace the parameter $r_{\infty}$ in them with $\tilde{r}_{\infty}$, we convert them into the forms

$$
\begin{aligned}
\tilde{\Sigma}_{i} & =\frac{1}{2} \tilde{r}_{\infty}^{2}\left(\tilde{r}_{\infty}^{2}+\tilde{r}_{s}^{2}\right), \quad \tilde{\Delta}_{i}=\frac{1}{2}\left(\tilde{r}_{\infty}^{2}-m\right)\left(\tilde{r}_{\infty}^{2}+\tilde{r}_{s}^{2}\right), \\
\tilde{\mathcal{K}} & =\frac{2\left(\tilde{r}_{\infty}^{2}-m\right)\left(\tilde{r}_{\infty}^{2}+\tilde{r}_{s}^{2}\right)}{\left(2 r^{2}+2 a^{2}-\tilde{r}_{\infty}^{2}-\tilde{r}_{s}^{2}\right)^{2}}
\end{aligned}
$$

respectively. With the help of Eq. (3.2), the line element (2.1) can be further brought into the following form:

$\mathrm{d} \tilde{s}_{(5)}^{2}=\mathrm{d} s_{(5)}^{2}\left(\Delta_{i} \rightarrow \tilde{\Delta}_{i}, \Sigma_{i} \rightarrow \tilde{\Sigma}_{i}, \mathcal{K} \rightarrow \tilde{\mathcal{K}}\right)$.
This equivalent form for the squashed rotating KK black holes tells us that they are characterised by the three independent parameters $\left(m, a, \tilde{r}_{\infty}\right)$.

In the absence of the parameters $m$ and $a$, the metric (3.3) is smoothly transformed to the one for the $\mathrm{KK}$ monopole, which is described by the following five-dimensional Ricciflat metric:

$$
\begin{aligned}
\mathrm{d} s_{\text {mon }}^{2}= & \left.\mathrm{d} \tilde{s}_{(5)}^{2}\right|_{m, a=0} \\
= & -\mathrm{d} t^{2}+\frac{\mathrm{d} \rho^{2}}{V^{2}}+\frac{\rho^{2}}{V^{2}}\left(\mathrm{~d} \theta^{2}+\sin ^{2} \theta \mathrm{d} \phi^{2}\right) \\
& +\frac{\tilde{r}_{\infty}^{2} V^{2}}{4}(\mathrm{~d} \psi+\cos \theta \mathrm{d} \phi)^{2},
\end{aligned}
$$

where $V=\left(1+\frac{\tilde{r}_{\infty}}{2 \rho}\right)^{-1 / 2}$ and we have performed the coordinate transformation $r=\tilde{r}_{\infty} V$ to get the last equality. As $\rho \rightarrow \infty$, the KK monopole asymptotes to the spacetime (2.4). Consequently, its boundary matches with that of the squashed rotating KK black hole.

In terms of the flat reference background (2.8), one can obtain the ADT mass of the monopole presented by Eq. (3.4), which is read off as

$M_{\mathrm{mp}}=\frac{\pi}{4 G_{N}^{(5)}} \tilde{r}_{\infty}^{2}$.

The mass $M_{\mathrm{mp}}$ agrees precisely with the one via the counterterm method [47] and satisfy the first law of black hole thermodynamics $\mathrm{d} M_{\mathrm{mp}}=I_{m p} \mathrm{~d} L_{\infty}$ and $2 M_{\mathrm{mp}}=I_{m p} L_{\infty}$ by virtue of the gravitational tension $I_{\mathrm{mp}}=\tilde{r}_{\infty} /\left(4 G_{N}^{(5)}\right)$ $[24,25]$. Here the gravitational tension $I_{\mathrm{mp}}$, as well as $\mathcal{I}$, is evaluated through the counterterm method [47] or the method based on the Hamiltonian formalism [53]. Actually, they can also be computed in the ADT formalism. As was shown in Refs. $[37,40]$, within the context of the Einstein gravity theory described by the Lagrangian $\mathfrak{L}_{\mathrm{gr}}=\sqrt{-g} R$, the ADT formalism completely agrees with the ADM one. Hence the gravitational tensions defined by using both formalisms coincide. That is to say, the expression for the gravitational tension based on the ADT formulation takes the same form as the ADM-type formula [54-57] given by Eq. (3.2) or Eq. (3.4) in [53]. Further making use of this expression to compute the gravitational tensions $I_{\mathrm{mp}}$ and $\mathcal{I}$, we obtain the same results as those in the counterterm method or the Hamiltonian approach.

Moreover, if the boundary matched KK monopole (3.4) is chosen as the reference background to compute the ADT mass $\tilde{M}$ of the five-dimensional squashed rotating KK black holes described by the line element in Eq. (2.1) or (3.3), we have

$$
\begin{aligned}
\tilde{M} & =M-M_{\mathrm{mp}} \\
& =\frac{\pi}{4 G_{N}^{(5)}}\left[\frac{\tilde{r}_{\infty}^{2}+m}{\tilde{r}_{\infty}}\left(\frac{\tilde{r}_{\infty}^{4}-4 m a^{2}}{\tilde{r}_{\infty}^{2}-m}\right)^{\frac{1}{2}}-\tilde{r}_{\infty}^{2}\right] .
\end{aligned}
$$


Here the mass $\tilde{M}$, which is still the desired thermodynamical quantity in fulfillment of the requirements for the first law of black hole thermodynamics

$$
\begin{aligned}
& \mathrm{d} \tilde{M}=T \mathrm{~d} S+\Omega_{H} \mathrm{~d} J_{\psi}+\tilde{\mathcal{I}} \mathrm{d} L_{\infty}, \\
& 2 \tilde{M}=3 T S+3 \Omega_{H} J_{\psi}+\tilde{\mathcal{I}} L_{\infty},
\end{aligned}
$$

where the gravitational tension computed on the KK monopole background $\tilde{\mathcal{I}}=\mathcal{I}-I_{\mathrm{mp}}$. Particularly, in the limit $r_{\infty} \rightarrow \infty, \tilde{\mathcal{I}}$ vanishes and the first law (3.7) becomes that of the usual five-dimensional black holes with two equal rotations. Furthermore, with the help of the gravitational tension $\tilde{\mathcal{I}}$, we establish the relation between the ADT mass $\tilde{M}$ and the Komar mass $M_{K}$ through the Legendre transformation $\tilde{M}=M_{K}+\tilde{W}_{\infty} \Xi_{\infty}$, where the new natural variable $\tilde{W}_{\infty}$ is given by $\tilde{W}_{\infty}=\tilde{\mathcal{I}}\left(2 c_{\psi} L_{\infty}\right)^{-1}=W_{\infty}-\left(16 \pi c_{\psi} G_{N}^{(5)}\right)^{-1}$, which differs from the variable $W_{\infty}$ only by a constant. In the light of $\tilde{W}_{\infty}$, the first law (2.13) relative to the Komar mass can be reexpressed in the alternative form

$\mathrm{d} M_{K}=T \mathrm{~d} S+\Omega_{H} \mathrm{~d} J_{\psi}-\Xi_{\infty} \mathrm{d} \tilde{W}_{\infty}$.

In comparison with the mass $M$ obtained on the asymptotical spacetime or the flat background, the mass $\tilde{M}$ has some remarkable features. First, if the two parameters $(m, a)$ are set zero, the squashed rotating KK black hole goes to its "empty" state, which is just the KK monopole (3.4). In this limit, $\tilde{M}$ vanishes, behaving like the mass of the Kerr-AdS black holes $[41,46]$ and the five-dimensional charged rotating Gödel-type black holes [48] in the absence of all the mass and rotation parameters, as well as the electric charge parameter. But $M \rightarrow M_{\mathrm{mp}}$ in the limit $(m, a) \rightarrow 0$, demonstrating that $M$ incorporates the mass of the KK monopole as its value in the "vacuum" state. Second, when $r_{\infty}$ goes to infinity, the metric (2.1) reduces to that of the ordinary five-dimensional rotating black holes with two equal angular momenta, while $\tilde{M}$ tends to the mass of these black holes at the same time, that is, $\tilde{M} \rightarrow 3 \pi m / 8$. However, in such a situation, $M$ is divergent. Third, the KK monopole (3.4), exactly satisfying the Einstein field equation in vacuum, is an on-shell reference background, and it has the same asymptotic structure as that of the squashed rotating KK black hole. By contrast, the flat background (2.8) fails to match the boundary of these black holes. As a consequence of the above-mentioned characters for the mass $\tilde{M}$, the boundary matched KK monopole may be a more natural reference background in the computation of the ADT mass.

In addition, comparing the ADT mass $\tilde{M}$ with the Komar mass $M_{K}$ that was evaluated without the requirement to preset any reference background, one observes that both of them behave consistently in the above-mentioned limits. Nevertheless, it should be advisable for us to distinguish $\tilde{M}$ from $M_{K}$ from the perspective of thermodynamics. As illustrated in Eqs. (3.7) and (3.8), there exist two different natural vari- ables $L_{\infty}$ and $\tilde{W}_{\infty}$ within the differential forms of the first law related to the ADT mass and the Komar one. According to this, $\tilde{M}$ and $M_{K}$ can be interpreted as thermodynamic potentials in thermodynamic environments described by different sets of natural variables. For $\tilde{M}$, the surroundings are described by the set of variables $\left(S, J_{\psi}, L_{\infty}\right)$ or $\left(S, J_{\psi}, \Xi_{\infty}\right)$, while those related to $M_{K}$ are characterised by $\left(S, J_{\psi}, \tilde{W}_{\infty}\right)$. At the end of this section, it should be pointed out that evaluations on the background (3.4) of the boundary matched KK monopole still give rise to the angular momentum $J_{\psi}$.

\section{The ADT mass under KK reduction}

In this section, thanks to the appearance of the compact direction $\psi$ in the metric (2.1) of the squashed rotating KK black hole, we may reconsider its mass by reducing the dimensions to four ones. If this is feasible, it will provide an effective approach to examining the mass obtained in five dimensions.

As a warm-up, let us briefly introduce the KK dimensional reduction for the Einstein gravity theory. Generally speaking, in the framework of the KK reduction theory, it is assumed that the $(D+1)$-dimensional solution of the Einstein gravity allows a compactified direction to exist so that the metric ansatz can be decomposed as $\mathrm{d} s_{(D+1)}^{2}=$ $e^{-2 \lambda \varphi /(D-1)} \mathrm{d} s_{(D)}^{2}+e^{\varphi / \lambda}(\mathrm{d} z+\mathcal{A})^{2}$, where the parameter $\lambda=-\sqrt{(D-1) / 2 /(D-2)}$ and all the $D$-dimensional fields $g_{\mu \nu}, \varphi$ and $\mathcal{A}_{\mu}$ are independent of the compactified direction $z$. Through a KK dimensional reduction along the $z$ direction, the higher-dimensional solution can be seen as the one in the context of the $D$-dimensional EinsteinMaxwell-dilaton theory with a particular dilaton coupling, whose Lagrangian has the following form:

$\mathfrak{L}_{\mathrm{EMD}}=\sqrt{-g}\left(R-\frac{1}{2} \nabla^{\mu} \varphi \nabla_{\mu} \varphi-\frac{1}{4} e^{2 \lambda \varphi} \mathcal{F}^{\mu \nu} \mathcal{F}_{\mu \nu}\right)$,

where the 2-form field strength for the KK gauge potential $\mathcal{A}_{v}$ is given by $\mathcal{F}_{\mu \nu}=2 \partial_{[\mu} \mathcal{A}_{\nu]}$. The equations of motion derived from the variation of the Lagrangian (4.1) with respect to the fields $\left(g_{\mu \nu}, \mathcal{A}_{\mu}\right)$ are given by

$$
\begin{aligned}
2 R_{\mu \nu} & =\left(\nabla_{\mu} \varphi\right)\left(\nabla_{\nu} \varphi\right)+e^{2 \lambda \varphi} \mathcal{F}_{\mu \sigma} \mathcal{F}_{\nu}{ }^{\sigma}-\frac{e^{2 \lambda \varphi} g_{\mu \nu} \mathcal{F}^{2}}{2(D-2)} \\
2 \nabla^{\mu} \nabla_{\mu} \varphi & =\lambda e^{2 \lambda \varphi} \mathcal{F}^{2}, \quad \nabla_{\mu}\left(e^{2 \lambda \varphi} \mathcal{F}^{\mu \nu}\right)=0
\end{aligned}
$$

Now, we can proceed with the explicit dimensional reduction of the line element (2.1) for the five-dimensional black hole with squashed horizons along the $\psi$ direction. After 
some computations, we obtain the four-dimensional solution that fully obeys the field equations (4.2) with the metric form

$$
\begin{aligned}
\mathrm{d} s_{(4)}^{2} & =-\frac{1}{4} \frac{\Delta \Sigma_{i}}{U \Delta_{i}} \mathrm{~d} t^{2}+\frac{r^{2} U \mathcal{K}^{2}}{\Delta} \mathrm{d} r^{2}+\frac{\mathcal{K} U}{4}\left(\mathrm{~d} \theta^{2}+\sin ^{2} \theta \mathrm{d} \phi^{2}\right), \\
\mathcal{A} & =-2 m a \omega \mathrm{d} t+\cos \theta \mathrm{d} \phi, \quad e^{2 \lambda \varphi}=\frac{U \Sigma}{4 H^{2}},
\end{aligned}
$$

where $U=\sqrt{H \Sigma} / 2$ and $\lambda=-\sqrt{3} / 2$ in the $D=4$ case. In the remainder of this section, we shall focus on evaluating the ADT mass of the squashed black holes in terms of the four-dimensional metric (4.2).

As is well known, within the context of the conventional ADT formalism, it is usually assumed that the matter fields, such as the gauge field and the scalar field, fall off very fast so that their contributions to the total conserved charges can be neglected. However, for the completeness and universality of this formalism, it is of great necessity to simultaneously take into consideration of the effects of the matter fields. In this work, we attempt to treat the gravitational field and the matter fields on an equal footing. For the Lagrangian (4.1), the potentials associated with the $\mathrm{U}(1)$ gauge field and the scalar field, derived in terms of the off-shell ADT method in Refs. $[38,40,42,43]$, are suitable for the desired ones of the fields $\mathcal{A}_{\mu}$ and $\varphi$ in the conventional ADT formalism. As a result, we directly present the total ADT potential $Q_{\mathrm{EMD}}^{\mu \nu}$ relevant to the Lagrangian (4.1) on the fixed reference background $\left(\bar{g}_{\mu \nu}, \overline{\mathcal{A}}_{\mu}, \bar{\varphi}\right)$ in the following form:

$$
\begin{aligned}
Q_{\mathrm{EMD}}^{\mu \nu}= & Q_{\mathrm{gr}}^{\mu \nu}+Q_{e d}^{\mu \nu}, \\
Q_{e d}^{\mu \nu}= & \frac{e^{2 \lambda \bar{\varphi}}}{4}\left[\vartheta\left(h \overline{\mathcal{F}}^{\mu \nu}+2 f^{\mu \nu}+4 h_{\sigma}^{[\mu} \overline{\mathcal{F}}^{v] \sigma}\right)+6 a_{\sigma} \xi^{[\sigma} \overline{\mathcal{F}}^{\mu \nu}\right] \\
& +\frac{e^{2 \lambda \bar{\varphi}}}{2 \lambda}\left(2 \lambda^{2} \vartheta \overline{\mathcal{F}}^{\mu \nu}-\xi^{[\mu} \bar{\nabla}^{\nu]} e^{-2 \lambda \bar{\varphi}}\right) \Delta \varphi,
\end{aligned}
$$

in which $\vartheta=\overline{\mathcal{A}}_{\sigma} \xi^{\sigma}$ and $f_{\mu \nu}=2 \partial_{[\mu} a_{\nu]}$, while the quantities $\left(a_{\mu}, \Delta \varphi\right)$ are defined through the perturbations of the matter fields $\left(\mathcal{A}_{\mu}, \varphi\right)$ around their background fields $\left(\overline{\mathcal{A}}_{\mu}, \bar{\varphi}\right)$, respectively, that is,

$a_{\mu}=\mathcal{A}_{\mu}-\overline{\mathcal{A}}_{\mu}, \quad \Delta \varphi=\varphi-\bar{\varphi}$,

rather than through the infinitesimal changes of the solution parameters like in the off-shell generalised ADT approach. In terms of the above potential, one is able to obtain the onshell ADT current through $J_{\mathrm{EMD}}^{\mu}=\bar{\nabla}_{\nu} Q_{\mathrm{EMD}}^{\mu \nu}$. It should be stressed that the background fields $\left(\bar{g}_{\mu \nu}, \overline{\mathcal{A}}_{\mu}, \bar{\varphi}\right)$ are required to fulfill the equations of motion in Eq. (4.2) in order to guarantee that the potential $Q_{\mathrm{EMD}}^{\mu \nu}$ fully inherits the spirit of the original ADT formulation. To the best of our knowledge, here the potential $Q_{\mathrm{EMD}}^{\mu \nu}$ might be the first one that naturally incorporates the contributions from the matter fields at the level of obeying the rules of the original ADT formalism.

With the help of the potential in Eq. (4.5), we are able to compute the mass of the five-dimensional squashed rotating KK black holes in terms of the four-dimensional gravitational field $g_{\mu \nu}^{(4)}$, the KK vector $\mathcal{A}_{\mu}$ and the dilaton $\varphi$ presented in Eq. (4.3) on appropriate reference spacetimes. In fact, by performing KK reduction, one can obtain two ones to achieve this. The first reference background, arising from the KK reduction of the flat background metric (2.8) along the $\psi$ direction, takes the form

$$
\begin{aligned}
\mathrm{d} \bar{s}_{(1)}^{2} & =\frac{\tilde{r}_{\infty}}{2}\left[-\mathrm{d} t^{2}+\mathrm{d} \rho^{2}+\rho^{2}\left(\mathrm{~d} \theta^{2}+\sin ^{2} \theta \mathrm{d} \phi^{2}\right)\right], \\
e^{2 \lambda \bar{\varphi}_{(1)}} & =\frac{\tilde{r}_{\infty}^{3}}{8}, \quad \overline{\mathcal{A}}_{(1)}=0 .
\end{aligned}
$$

After performing the coordinate transformation $r \rightarrow \rho$ in Eq. (2.3) to reexpress the four-dimensional reduced metric (4.3) into the form in the coordinate system $(t, \rho, \theta, \phi)$, we make use of the ADT potential $Q_{\mathrm{EMD}}^{\mu \nu}$ to evaluate the mass $M_{(1)}$ on the background metric (4.6) and obtain

$M_{(1)}=M$,

which precisely equals the mass evaluated on the fivedimensional flat reference spacetime in Eq. (2.9). To arrive at the above equation, one should bear in mind that the standard relation $G_{N}^{(5)}=4 \pi G_{N}^{(4)}$ has been employed.

On the other hand, we can also choose the following reference background:

$$
\begin{aligned}
\mathrm{d} \bar{s}_{(2)}^{2} & =\frac{\tilde{r}_{\infty}}{2}\left[-V \mathrm{~d} t^{2}+\frac{\mathrm{d} \rho^{2}}{V}+\frac{\rho^{2}}{V}\left(\mathrm{~d} \theta^{2}+\sin ^{2} \theta \mathrm{d} \phi^{2}\right)\right], \\
e^{2 \lambda \bar{\varphi}(2)} & =\frac{1}{8} \tilde{r}_{\infty}^{3} V^{3}, \quad \overline{\mathcal{A}}_{(2)}=\cos \theta \mathrm{d} \phi
\end{aligned}
$$

in the evaluation of the mass, which satisfies the field equations in Eq. (4.2) and coincides with the KK monopole (3.4) by lifting it back to five dimensions. From the perspective of four dimensions, calculating the ADT mass of the KK monopole (4.8) on the reference background (4.6), one obtains the same value $M_{\mathrm{mp}}$ as in Eq. (3.5). Furthermore, a complex calculation on the background metric (4.8) yields the ADT mass

$M_{(2)}=\tilde{M}$,

which is consistent with the mass got on the background of the boundary matched KK monopole.

A remark is in order here. Unlike the application of the usual ADT formulation, in addition to the contribution of the gravitational field, those from the matter fields, such as the KK gauge potential $\mathcal{A}_{\mu}$ and the dilaton $\varphi$, have been taken into account in the evaluation of the mass $M_{(1)}$ and $M_{(2)}$ within the framework of the four-dimensional reduced theory. However, it can be observed that they actually make no contributions since the KK vector decreases quite quickly so that its $t$-component $\mathcal{A}_{t} \rightarrow 0$ and the dilatons $e^{2 \lambda \varphi}=$ $e^{2 \lambda \bar{\varphi}_{(1)}}=e^{2 \lambda \bar{\varphi}_{(2)}}$ when the radial coordinate $\rho \rightarrow \infty$, giving rise to the vanishing of the $(t, r)$-component of the potential $Q_{e d}^{\mu \nu}$. To this point, there is only a need to compute the 
potential $Q_{\mathrm{gr}}^{\mu \nu}$ with respect to the gravitational field for the sake of obtaining the ADT mass of the rotating black holes with squashed horizons. As a matter of fact, one can evaluate the mass $M_{(1)}$ on the on-shell reference background of the four-dimensional Minkowski metric

$\mathrm{d} \bar{s}_{4 d \text { flat }}^{2}=-\mathrm{d} t^{2}+\mathrm{d} \rho^{2}+\rho^{2}\left(\mathrm{~d} \theta^{2}+\sin ^{2} \theta \mathrm{d} \phi^{2}\right)$.

Due to the above reference metric in the dimensionally reduced theory, one is able to see that the extra dimension in the asymptotic geometry (2.4) and the flat background (2.8) actually makes no contribution to the ADT mass. Hence calculations on the two backgrounds yield the same value $M$.

\section{Mass via the off-shell ADT approach}

In this section, to compare with the original ADT formulation, we shall make use of its off-shell generalisation ${ }^{1}$ recently proposed in [37] and developed in [38-40] to compute the mass of the squashed rotating KK black holes. In contrast to the original ADT formalism, the off-shell generalised one possesses the dramatically different feature that all the fields are linearised about arbitrary background fields.

According to the off-shell ADT formalism, the perturbations of the gravitational field are induced by those of the solution parameters, such as $m, a$ and $r_{\infty}$. However, it is worth noting that one cannot naively fix the parameter $r_{\infty}$ but merely lets the parameters $(m, a)$ vary to fluctuate the gravitational field, as the case of the static black holes with squashed horizons in [28]. Otherwise, the conserved charge will suffer from the non-integrable problem. Therefore, in order to avoid such a dilemma, we find that a feasible approach is to adopt the metric form (3.3) instead of the one (2.1) as well as to make the parameter $\tilde{r}_{\infty}$ rather than $r_{\infty}$ be fixed in the evaluation of the mass. That is to say, the perturbations of the metric form (3.3) are only determined by the infinitesimal variation of the parameters $(m, a)$ through

$m \rightarrow m+\mathrm{d} m, \quad a \rightarrow a+d a$.

Considering the pattern for the fluctuations of the gravitational field induced from Eq. (5.1), we employ Eq. (2.6) to compute the off-shell ADT charge $\mathcal{Q}\left[\xi_{(t)}^{\mu}\right]$ associated with the timelike Killing vector $\xi_{(t)}^{\mu}=(-1,0,0,0,0)$ and obtain

$\mathrm{d} \mathcal{Q}\left[\xi_{(t)}^{\mu}\right]=\frac{M}{2}\left(\frac{\left(3 \tilde{r}_{\infty}^{2}-m\right) \mathrm{d} m}{\tilde{r}_{\infty}^{4}-m^{2}}-\frac{4 d\left(m a^{2}\right)}{\tilde{r}_{s}^{4}}\right)$,

\footnotetext{
${ }^{1}$ Before Ref. [37], in [52], the form of the current has the potential to construct an off-shell ADT current in the context of pure gravity theories.
}

whose integral yields the mass $\mathcal{M}$,

$\mathcal{M}=\tilde{M}$.

Here the mass $\mathcal{M}$ is in agreement with the one got on the reference spacetime of the KK monopole in Eq. (3.6). As in the case for the static squashed black holes [28], this may result from the manner of parameterising the space of solutions. Actually, according to the formula of the conserved charges via the off-shell generalised ADT method, the lower limit of the integration for the solution parameters $(m, a)$ indicates that there exists a "hidden" reference background that is just the KK monopole given by Eq. (3.4). Besides, to guarantee the integrability of the mass $\mathcal{M}$, it is desired that the parameter $\tilde{r}_{\infty}$ be unchangeable, which implies that this parameter is not able to be treated as a thermodynamical variable from the viewpoint of the off-shell ADT method and the KK monopole characterised by $\tilde{r}_{\infty}$ should be a fixed background. Hence, due to the above-mentioned, it might be quite appropriate to choose the boundary matched KK monopole as the reference spacetime of the squashed rotating KK black holes when the original ADT formalism is applied.

Of course, the off-shell generalised ADT method can also be applied to compute the mass of the rotating black holes with squashed horizons under KK reduction along the compactified direction. As shown in Ref. [40], one will find that the mass remains the same before and after the dimensional reduction. Moreover, evaluating the angular momentum in terms of the off-shell generalised ADT method, one will find that it is consistent with the value $J_{\psi}$ of the angular momentum in the original ADT formalism.

\section{Summary}

In the present work, apart from the asymptotical geometry (2.4), which was adopted as the common reference background in the evaluation of the ADT mass for the fivedimensional rotating black holes with squashed horizons in $[3,26]$, we have found that there exist another two reference backgrounds that are appropriate for the calculations of the ADT mass of these black holes. They are the flat background (2.8) and the boundary matched KK monopole (3.4). Both of them satisfy the five-dimensional vacuum Einstein equation, fulfilling the on-shell requirement of the reference background in the original ADT formalism, but the off-shell asymptotical geometry breaks down. Since the squashed rotating KK black hole (3.3) coincides with the KK monopole (3.4) in the limit $(m, a) \rightarrow(0,0)$ and they have the same boundaries, it is natural to choose the KK monopole as the on-shell reference background.

Evaluating the mass on the background spacetimes (2.8) and (3.4), we obtained the values $M$ and $\tilde{M}$ given by Eqs. (2.9) and (3.6) respectively, which differ from each other by 
the mass of the KK monopole $M_{\mathrm{mp}}$ and satisfy the first law with the same natural variables. Nevertheless, since $\tilde{M}$ vanishes in the limit $(m, a) \rightarrow(0,0)$ and it becomes that of the usual five-dimensional neutral black holes with two equal angular momenta in the limit $\tilde{r}_{\infty} \rightarrow \infty$, we propose that it might be better to choose $\tilde{M}$ as the ADT mass of the squashed rotating KK black holes. On the other hand, in spite of the fact that both the mass $\tilde{M}$ and the Komar one $M_{K}$ behave consistently in such limits, we have demonstrated that they can be seen as different thermodynamic potentials with different sets of natural variables due to the expressions of the first law. However, in order to go deep into study of the relation between the masses as well as the physical interpretation of the reference backgrounds, it may be deserved to investigate other aspects of the squashed rotating KK black holes along the lines of [25] from the thermodynamical perspective, such as the calculations of the free energy and Hamiltonian on different backgrounds and the analyses of the thermodynamic stability in various environments.

For the sake of examining the ADT mass in five dimensions, with the help of the KK reduction theory, we recomputed it from the perspective of the dimensionally reduced theory. Within the context of the four-dimensional reduced theory, to take into consideration of the contributions from the KK vector and the dilaton, we modified the potential by strictly following the rules of the conventional ADT method. In terms of this potential to calculate the mass $M_{(1)}$ and $M_{(2)}$, we found that they are in agreement with the fivedimensional mass $M$ and $\tilde{M}$, respectively. Furthermore, as comparison, the off-shell generalised ADT formulation was applied to compute the mass of the squashed rotating KK black holes, yielding the value $\tilde{M}$. This supports that the boundary matched KK monopole should be adopted as the reference background in the context of the original ADT formalism.

Finally, it should be emphasised that the analysis in this work can be successfully extended to the charged generalisations of the neutral rotating KK black holes found in [5] although we merely deal with the ADT mass of the neutral ones. However, the applications to the more general squashed KK black holes, for instance, the charged rotating Gödel-type black holes [6] and the three-charge rotating black holes with squashed horizons [7], need to be checked in the future work. If the physical charges of these black holes can be obtained, we will take an important step towards understanding the universality for the reference backgrounds of the flat background and the boundary matched KK monopole.

Acknowledgements The author thanks the anonymous referees for their valuable suggestions. This work was supported by the Natural Science Foundation of China under Grant no. 11505036 and No. 11275157. It was also partially supported by the Technology Department of Guizhou province Fund under Grant no. (2016)1104 and the
Guizhou province science and technology innovation talent team [Grant no. (2015)4015].

Open Access This article is distributed under the terms of the Creative Commons Attribution 4.0 International License (http://creativecomm ons.org/licenses/by/4.0/), which permits unrestricted use, distribution, and reproduction in any medium, provided you give appropriate credit to the original author(s) and the source, provide a link to the Creative Commons license, and indicate if changes were made.

Funded by $\mathrm{SCOAP}^{3}$.

\section{References}

1. H. Ishihara, K. Matsuno, Prog. Theor. Phys. 116, 417 (2006)

2. S.S. Yazadjiev, Phys. Rev. D 74, 024022 (2006)

3. T. Wang, Nucl. Phys. B 756, 86 (2006)

4. S. Tomizawa, A. Ishibashi, Class. Quantum Grav. 25, 245007 (2008)

5. T. Nakagawa, H. Ishihara, K. Matsuno, S. Tomizawa, Phys. Rev. D 77, 044040 (2008)

6. S. Tomizawa, H. Ishihara, K. Matsuno, T. Nakagawa, Prog. Theor. Phys. 121, 823 (2009)

7. S. Tomizawa. arXiv: 1009.3568 [hep-th]

8. M. Allahverdizadeh, K. Matsuno, Phys. Rev. D 81, 044001 (2010)

9. Y. Brihaye, E. Radu, C. Stelea, Phys. Lett. B 709, 293 (2012)

10. Y. Brihaye, E. Radu, Phys. Lett. B 641, 212 (2006)

11. L.Y. Ji, S.B. Chen, J.L. Jing, J. High Energy Phys. 03, 089 (2014)

12. J. Sadeghi, J. Naji, H. Vaez, Phys. Lett. B 728, 170 (2014)

13. K. Matsuno, H. Ishihara, Phys. Rev. D 80, 104037 (2009)

14. R. Nishikawa, M. Kimura, Class. Quantum Grav. 27, 215020 (2010)

15. M. Kimura, K. Murata, H. Ishihara, J. Soda, Phys. Rev. D 77, 064015 (2008)

16. S. Tomizawa, Phys. Rev. D 82, 104047 (2010)

17. X. He, B. Wang, S.B. Chen, Phys. Rev. D 79, 084005 (2009)

18. M.M. Stetsko, Eur. Phys. J. C 76, 48 (2016)

19. K. Matsuno, K. Umetsu, Phys. Rev. D 83, 064016 (2011)

20. S.W. Wei, R. Li, Y.X. Liu, J.R. Ren, Eur. Phys. J. C 65, 281 (2010)

21. S.B. Chen, B. Wang, R.K. Su, Phys. Rev. D 77, 024039 (2008)

22. H. Ishihara, J. Soda, Phys. Rev. D 76, 064022 (2007)

23. R.G. Cai, L.M. Cao, N. Ohta, Phys. Lett. B 639, 354 (2006)

24. Y. Kurita, H. Ishihara, Class. Quantum Grav. 24, 4525 (2007)

25. Y. Kurita, H. Ishihara, Class. Quantum Grav. 25, 085006 (2008)

26. J.J. Peng, S.Q. Wu, Nucl. Phys. B 828, 273 (2010)

27. S.Q. Wu, D. Wen, Q.Q. Jiang, S.Z. Yang, Phys. Lett. B 726, 404 (2013)

28. J.J. Peng, W.C. Xiang, S.H. Cai, Chin. Phys. Lett. 33, 080401 (2016)

29. X.D. Zhu, D. Wu, S.Q. Wu, S.Z. Yang, Gen. Rel. Grav. 48, 154 (2016)

30. C. Stelea, K. Schleich, D. Witt, Phys. Rev. D 78, 124006 (2008)

31. M. Saito, H. Noda, T. Tashiro, Int. J. Mod. Phys. A 24, 2357 (2009)

32. L.F. Abbott, S. Deser, Nucl. Phys. B 195, 76 (1982)

33. L.F. Abbott, S. Deser, Phys. Lett. B 116, 259 (1982)

34. S. Deser, B. Tekin, Phys. Rev. Lett. 89, 101101 (2002)

35. S. Deser, B. Tekin, Phys. Rev. D 67, 084009 (2003)

36. S. Deser, B. Tekin, Phys. Rev. D 75, 084032 (2007)

37. W. Kim, S. Kulkarni, S.H. Yi, Phys. Rev. Lett. 111, 081101 (2013)

38. S. Hyun, J. Jeong, S.A. Park, S.H. Yi, Phys. Rev. D 90, 104016 (2014)

39. J.J. Peng, Eur. Phys. J. C 74, 3156 (2014)

40. J.J. Peng, Phys. Rev. D 95, 104022 (2017)

41. Y.D. Jing, J.J. Peng, Chin. Phys. B 26, 100401 (2017)

42. M.R. Setare, H. Adami, Class. Quantum Grav. 34, 105008 (2017) 
43. J.J. Peng, Int. J. Mod. Phys. A 31, 1650060 (2016)

44. G. Barnich, F. Brandt, Nucl. Phys. B 633, 3 (2002)

45. G. Barnich, Class. Quantum Grav. 20, 3685 (2003)

46. G. Barnich, G. Compere, Phys. Rev. D 71, 044016 (2005) [Erratum: Phys. Rev. D 73, 029904 (2006)]

47. R.B. Mann, C. Stelea, Phys. Lett. B 634, 531 (2006)

48. S.Q. Wu, Phys. Rev. Lett. 100, 121301 (2008)

49. P. Dobiasch, D. Maison, Gen. Rel. Grav. 14, 231 (1982)

50. D. Pollard, J. Phys. A: Math. Gen. 16, 565 (1983)
51. G.W. Gibbons, D.L. Wiltshire, Ann. Phys. 167, 201 (1986) [Erratum: Ann. Phys. 176, 393 (1987)]

52. A. Bouchareb, G. Clement, Class. Quantum Grav. 24, 5581 (2007)

53. T. Harmark, N.A. Obers, J. High Energy Phys. 05, 043 (2004)

54. J.H. Traschen, D. Fox, Class. Quantum Grav. 21, 289 (2004)

55. P.K. Townsend, M. Zamaklar, Class. Quantum Grav. 18, 5269 (2001)

56. T. Harmark, N.A. Obers, Class. Quantum Grav. 21, 1709 (2004)

57. B. Kol, E. Sorkin, T. Piran, Phys. Rev. D 69, 064031 (2004) 